\title{
LIOUVILLE THEOREMS FOR THE POLYHARMONIC HÉNON-LANE-EMDEN SYSTEM*
}

\author{
MOSTAFA FAZLY†
}

\begin{abstract}
We study Liouville theorems for the following polyharmonic Hénon-Lane-Emden system

$$
\left\{\begin{aligned}
(-\Delta)^{m} u & =|x|^{a} v^{p} \text { in } \mathbb{R}^{n} \\
(-\Delta)^{m} v & =|x|^{b} u^{q} \text { in } \mathbb{R}^{n}
\end{aligned}\right.
$$

when $m, p, q \geq 1, p q \neq 1, a, b \geq 0$. The main conjecture states that $(u, v)=(0,0)$ is the unique nonnegative solution of this system whenever $(p, q)$ is under the critical Sobolev hyperbola, i.e. $\frac{n+a}{p+1}+\frac{n+b}{q+1}>n-2 m$. We show that this is indeed the case in dimension $n=2 m+1$ for bounded solutions. In particular, when $a=b$ and $p=q$, this means that $u=0$ is the only nonnegative bounded solution of the polyharmonic Hénon equation

$$
(-\Delta)^{m} u=|x|^{a} u^{p} \text { in } \mathbb{R}^{n}
$$

in dimension $n=2 m+1$ provided $p$ is the subcritical Sobolev exponent, i.e., $1<p<1+4 m+2 a$. Moreover, we show that the conjecture holds for radial solutions in any dimensions. It seems the power weight functions $|x|^{a}$ and $|x|^{b}$ make the problem dramatically more challenging when dealing with nonradial solutions.
\end{abstract}

Key words. Henon-Lane-Emden system, Liouville theorems, entire solutions, polyharmonic semilinear elliptic equations.

AMS subject classifications. 35J61,35B08, 35B53, 35A23, 35A01.

1. Introduction and main results. We examine the following weighted system known as the polyharmonic Hénon-Lane-Emden system

$$
\left\{\begin{aligned}
(-\Delta)^{m} u & =|x|^{a} v^{p} \text { in } \mathbb{R}^{n} \\
(-\Delta)^{m} v & =|x|^{b} u^{q} \text { in } \mathbb{R}^{n}
\end{aligned}\right.
$$

where $m, p, q \geq 1, p q \neq 1, a, b \geq 0$. This is the statement of the Hénon-Lane-Emden conjecture for polyharmoic system (1).

Conjecture 1. Let $(u, v)$ be a nonnegative solution of system (1). Suppose $(p, q)$ is under the critical hyperbola, i.e.,

$$
\frac{n+a}{p+1}+\frac{n+b}{q+1}>n-2 m
$$

Then $u=v=0$.

Note that it is very straightforward to give a positive answer to Conjecture 1 in all dimensions $1 \leq n \leq 2 m$. This is in fact a quick consequence of $L^{1}$ estimates given in Lemma 1. Therefore, in this paper, we focus on higher dimensions $n \geq 2 m+1$.

Liouville theorems for system (1) are very widely studied for the past few decades. In what follows we briefly review some of the related known results. We divide the introduction into two different cases. We first consider the case $a=b=0$ and then

\footnotetext{
${ }^{*}$ Received July 23, 2013; accepted for publication October 10, 2013.

$\dagger$ Department of Mathematical and Statistical Sciences, University of Alberta, Edmonton, Alberta, Canada T6G 2G1 (fazly@ualberta.ca). The author is pleased to acknowledge the support of a University of Alberta start-up grant and a discovery grant from National Sciences and Engineering Research Council of Canada (NSERC).
} 
the other case that is when one of the parameters $a$ or $b$ is not zero. In this paper, we mainly focus on system (1) whenever there are weight functions $|x|^{a}$ and $|x|^{b}$. As a matter of fact, the weight functions make the problem much more challenging and as a general statement, some standard techniques such as moving plane methods and certain Sobolev embeddings cannot be applied anymore.

1.1. The case $a=b=0$. System (1) when $a=b=0$ is well studied and there are many interesting results on classifying the solutions of this system for various $p$ and $q$.

We begin by the scalar case that is when $p=q>1$. For the Lane-Emden equation (i.e., when $m=1, p=q>1$ and $a=b=0$ ) a celebrated theorem by Gidas-Spruck $[9,10]$ states that there is no positive solution for the Lane-Emden equation

$$
-\Delta u=u^{p} \text { in } \mathbb{R}^{n}
$$

whenever $1<p<\frac{n+2}{n-2}$ for $n \geq 3$. This Liouville theorem is optimal as shown by Gidas, Ni and Nirenberg in [8] under the assumption that $u=O\left(|x|^{2-n}\right)$, and by Caffarelli, Gidas and Spruck in [2] without the growth assumption. See also Chen and $\mathrm{Li}[4]$ for an easier proof based on the moving planes method. In the case of the fourth order Lane-Emden equation (i.e., when $m=2, p=q>1$ and $a=b=0$ ) and the polyharmonic Lane-Emden equation (i.e., when $m \geq 1, p=q>1$ and $a=b=0$ )

$$
(-\Delta)^{m} u=u^{p} \quad \text { in } \mathbb{R}^{n}
$$

similar Liouville theorems are proved by Lin [13] and Wei and $\mathrm{Xu}$ in [29] for the subcritical Sobolev exponent that is $1<p<\frac{n+2 m}{n-2 m}, n>2 m$. Note that this exponent appears in the Sobolev embedding $W^{m, 2} \hookrightarrow L^{p}$.

Now we focus on the case that the parameters $p$ and $q$ are not necessarily equal. Therefore, we are dealing with a system of equations. This case is much less understood than the scalar case. For the Lane-Emden system (i.e., when $m=1, a=b=0$ and $p, q \geq 1$ when $p q \neq 1$ )

$$
\left\{\begin{array}{l}
-\Delta u=v^{p} \text { in } \mathbb{R}^{n} \\
-\Delta v=u^{q} \text { in } \mathbb{R}^{n}
\end{array}\right.
$$

Conjecture 1 is known as the Lane-Emden conjecture and the curve $\frac{1}{p+1}+\frac{1}{q+1}=\frac{n-2}{n}$ is the critical Sobolev hyperbola. Proving such a nonexistence result seems to be very challenging problem. However, there are many interesting papers that cover certain dimensions. The case of radial solutions was solved by Mitidieri [15] in any dimension, and both Mitidieri [15] and Serrin-Zou [24] constructed positive radial solutions on and above the critical hyperbola, i.e. $\frac{1}{p+1}+\frac{1}{q+1} \leq \frac{n-2}{n}$, which means that the nonexistence theorem is optimal for radial solutions. For nonradial solutions of the Lane-Emden system, certain Liouville theorems are proved for various parameters $p$ and $q$ by Souto in [27], Mitidieri in [15] and Serrin-Zou in [23], that in a particular case they give a positive answer to the Lane-Emden conjecture in dimensions $1 \leq n \leq 2$. In dimension $n=3$, Serrin-Zou [23] gave a proof for the nonexistence of polynomially bounded solutions, an assumption that was relaxed later by Poláčik, Quittner and Souplet [21]. See also [1, 7]. More recently, Souplet [26] completely settled the conjecture in dimension $n=4$, while providing in dimensions $n \geq 5$, a more restrictive new region for the exponents $(p, q)$ that insures nonexistence. The Lane-Emden conjecture is an open problem for dimensions $n \geq 5$. For the polyharmonic case $m \geq 1$, it is known 
that $\frac{1}{p+1}+\frac{1}{q+1}=\frac{n-2 m}{n}$ is the critical Sobolev hyperbola. Conjecture 1 for the case of radial solutions was solved by Liu et. al. in [14] in any dimensions and as far as we know only some partial results are given for the nonradial solutions in [14, 28, 12]. Note that Caristi, DAmbrosio and Mitidieri in [3] have proved Liouville theorems for supersolutions of system (1) and also they have explored the connection between (1) and the Hardy-Littlewood-Sobolev systems (HLS).

1.2. The case $a \neq 0$ and or $b \neq 0$. The power weight function has been of interest in this context and it was introduced by M. Hénon [11] in equation

$$
\begin{cases}-\Delta u=|x|^{a} u^{p} & \text { for }|x|<1 \\ u=0 & \text { on }|x|=1,\end{cases}
$$

to model and study spherically symmetric clusters of stars. This equation is now known as the Hénon equation for $a>0$ and the Hénon-Hardy equation for $a<0$. Ten years later, Ni in [18] explored properties of positive radial solutions of the Hénon equation on the unit ball and observed the fact that the power profile $|x|^{a}$ enlarges considerably the range of solvability beyond the classical critical threshold, i.e., $p<$ $2^{*}-1=\frac{n+2}{n-2}$ to $p<2^{*}-1+\frac{2 a}{n-2}=\frac{n+2+2 a}{n-2}$ where $2^{*}$ is the critical Sobolev exponent for the Sobolev embedding $W^{1,2} \hookrightarrow L^{p}$. On the other hand, as it is shown by Smets, Su and Willem in [25] and references therein, equation (3) also admits nonradial solutions for $p<2^{*}-1$. The existence of nonradial solutions for the full range $p<\frac{n+2+2 a}{n-2}$ is still an open problem. Note that since the function $|x| \rightarrow|x|^{a}$ is increasing, the classical moving planes arguments given by Gidas, $\mathrm{Ni}$ and Nirenberg in [8] cannot be applied to prove the radial symmetry of the solutions of (3). Therefore, the existence of nonradial solutions for this equation is natural and it is studied in many interesting papers.

Regarding Liouville theorems, Phan and Souplet [20] for the first time attacked the problem and showed among other results that conjecture for the scalar case, that is $m=1$ and $a=b$ and $p=q>1$, holds for bounded nonnegative solutions in dimension $n=3$. Here is the result,

Theorem A. (Phan-Souplet [20]) Let $n=3, m=1, a=b>-2$ and $p=q>1$. Assume $(p, q)$ satisfies (2) that is $1<p<5+2 a$, then there is no positive bounded solution for the Hénon equation, i.e.,

$$
-\Delta u=|x|^{a} u^{p} \quad \text { in } \mathbb{R}^{n}
$$

For the case of systems (1) when $m=1$, the author with Ghoussoub in [6] have also proved the conjecture in dimension three for bounded solutions. See also [19] for dimensions three and some partial results for dimension four. In this note, we shall first extend the result of Fazly-Ghoussoub [6] and Phan-Souplet [20] to the full polyharmonic Hénon-Lane-Emden system by showing the following.

THEOREM 1. Conjecture 1 holds in dimension $n=2 m+1$ for nonnegative bounded solutions of (1).

THEOREM 2. Conjecture 1 holds in all dimensions for nonnegative radial solutions of (1).

For the special case $p=q$ and $a=b$, we have the following weighted equation known as the polyharmonic Hénon equation

$$
(-\Delta)^{m} u=|x|^{a} u^{p} \text { in } \mathbb{R}^{n},
$$


where $p>1$ and $a \geq 0$. Note that under the critical hyperbola (2) turns into the following subcritical Sobolev exponent

$$
1<p<\frac{n+2 m+2 a}{n-2 m} \text { where } n>2 m \text {. }
$$

As a consequence of Theorem $1, u=0$ is the unique nonnegative bounded solution of (5) in dimension $n=2 m+1$ provided (6) holds that is $1<p<1+4 m+2 a$. Also, Theorem 2 implies that $u=0$ is the unique nonnegative radial solution of (5) provided (6) holds in all dimensions. Let us mention that very recently, Cowan in [5] following ideas developed in [20,6] considered the fourth order Hénon equation, that is (5) when $m=2$, and proved that in dimension five there is no bounded positive solution for (5) provided $1<p<9+2 a$.

Here is the organization of the paper. In Section 2, we prove Theorem 1 via applying various methods developed in the theory of elliptic regularity. In Section 3, we prove Theorem 2 via certain ODE arguments. Our methods of proof are strongly motivated by the ideas developed by Souplet in [26], Phan-Souplet in [20], Mitidieri et. al. in [16, 15, 17], Wei-Xu in [29], Fazly-Ghoussoub in [6] and references therein.

2. Liouville theorems for nonradial solutions via elliptic estimates. We start with the following standard $L^{1}\left(B_{R}\right)$ estimate on the right hand side of system (1). Similar estimates for the second order case are given in [17, 22, 23].

LEMMA 1. For any nonnegative entire solution $(u, v)$ of (1) and $R>1$, there holds

$$
\begin{aligned}
& \int_{B_{R}}|x|^{a} v^{p} \leq C R^{n-2 m-\frac{(b+2 m) p+(a+2 m)}{p q-1}}, \\
& \int_{B_{R}}|x|^{b} u^{q} \leq C R^{n-2 m-\frac{(a+2 m) q+(b+2 m)}{p q-1}},
\end{aligned}
$$

where the positive constant $C=C(n, m, t, s, a, b, p, q)$ does not depend on $R$.

Note that $(7)$ and (8) imply that if $(u, v)$ is a nonnegative entire solution of (1) then $u=v=0$ in dimensions

$$
n-2 m<\max \left\{\frac{(b+2 m) p+(a+2 m)}{p q-1}, \frac{(a+2 m) q+(b+2 m)}{p q-1}\right\} .
$$

In particular this proves Conjecture 1 in dimensions $1 \leq n \leq 2 m$. However this does not cover under the critical hyperbola mentioned in Conjecture 1 for $n \geq 2 m+1$. In other words, (2) is equivalent to the following when $p q>1$

$$
n-2 m<\frac{(b+2 m)(p+1)+(a+2 m)(q+1)}{p q-1} .
$$

Proof. Fix the following standard test function $\phi_{R} \in C_{c}^{\infty}\left(\mathbb{R}^{n}\right)$ when $0 \leq \phi_{R} \leq 1$ and

$$
\phi_{R}(x)= \begin{cases}1, & \text { if }|x|<R \\ 0, & \text { if }|x|>2 R\end{cases}
$$

where $\left\|D_{x}^{i} \phi_{R}\right\|_{L^{\infty}\left(\mathbf{R}^{n}\right)} \leq \frac{C}{R^{i}}$ for $i=1, \cdots, 2 m$. For any $t \geq 2 m$, we have

$$
\left|\Delta^{m} \phi_{R}^{t}(x)\right| \leq C \begin{cases}0, & \text { if }|x|<R \text { or }|x|>2 R ; \\ R^{-2 m} \phi_{R}^{t-2 m}, & \text { if } R<|x|<2 R .\end{cases}
$$


Now test the first equation of (1) by $\phi_{R}^{t}$ and integrate to get

$$
\int_{B_{2 R}}|x|^{a} v^{p} \phi_{R}^{t} \leq C R^{-2 m} \int_{B_{2 R} \backslash B_{R}} u \phi_{R}^{t-2 m} .
$$

Applying the Hölder's inequality we get

$$
\int_{B_{2 R}}|x|^{a} v^{p} \phi_{R}^{t} \leq C R^{\left(n-\frac{b}{q} q^{\prime}\right) \frac{1}{q^{\prime}}-2 m}\left(\int_{B_{2 R} \backslash B_{R}}|x|^{b} u^{q} \phi_{R}^{(t-2 m) q}\right)^{1 / q} .
$$

By a similar calculation for $s \geq 2 m$, we obtain

$$
\int_{B_{2 R}}|x|^{b} u^{q} \phi_{R}^{s} \leq C R^{\left(n-\frac{a}{p} p^{\prime}\right) \frac{1}{p^{\prime}}-2 m}\left(\int_{B_{2 R} \backslash B_{R}}|x|^{a} v^{p} \phi_{R}^{(s-2 m) p}\right)^{\frac{1}{p}}
$$

where $\frac{1}{p}+\frac{1}{p^{\prime}}=1$. Since $p q>1$, for large enough $s$ we have $2 m+\frac{s}{q}<(s-2 m) p$. So, we can choose $t$ such that $2 m+\frac{s}{q} \leq t \leq(s-2 m) p$ which means that $t \leq(s-2 m) p$ and $s \leq(t-2 m) q$. Therefore, $\phi_{R}^{(t-2 m) q} \leq \phi_{R}^{s}$ and $\phi_{R}^{(s-2 m) p} \leq \phi_{R}^{t}$. Now, by collecting the above inequalities for $p q>1$ we get

$$
\begin{aligned}
\left(\int_{B_{2 R}}|x|^{a} v^{p} \phi_{R}^{t}\right)^{p q} & \leq C R^{\left[\left(n-\frac{b}{q} q^{\prime}\right) \frac{1}{q^{\prime}}-2 m\right] p q}\left(\int_{B_{2 R} \backslash B_{R}}|x|^{b} u^{q} \phi_{R}^{s}\right)^{p} \\
& \leq C R^{(n-2 m)(p q-1)-[(b+2 m) p+(a+2 m)]} \int_{B_{2 R} \backslash B_{R}}|x|^{a} v^{p} \phi_{R}^{t}
\end{aligned}
$$

and

$$
\begin{aligned}
\left(\int_{B_{2 R}}|x|^{b} u^{q} \phi_{R}^{s}\right)^{p q} & \leq C R^{\left[\left(n-\frac{a}{p} p^{\prime}\right) \frac{1}{p^{\prime}}-2 m\right] p q}\left(\int_{B_{2 R} \backslash B_{R}}|x|^{a} v^{p} \phi_{R}^{t}\right)^{q} \\
& \leq C R^{(n-2 m)(p q-1)-[(a+2 m) q+(b+2 m)]} \int_{B_{2 R} \backslash B_{R}}|x|^{b} u^{q} \phi_{R}^{s} .
\end{aligned}
$$

By using the Hölder's inequality, we can now get the following interpolation estimates on $u$ and $v$.

COROLlary 1. With the same assumptions as Lemma 1, the following holds.

(i) For any $0<t<q$ and any $0<s<p$

$$
\int_{B_{R} \backslash B_{R / 2}} v^{s} \leq C R^{n-\frac{(a+2 m) q+(b+2 m)}{p q-1} s} \text { and } \int_{B_{R} \backslash B_{R / 2}} u^{t} \leq C R^{n-\frac{(b+2 m) p+(a+2 m)}{p q-1} t} .
$$

(ii) For any $0<t<\frac{n q}{n+b}$ and any $0<s<\frac{n p}{n+a}$

$$
\int_{B_{R}} v^{s} \leq C R^{n-\frac{(a+2 m) q+(b+2 m)}{p q-1} s} \text { and } \int_{B_{R}} u^{t} \leq C R^{n-\frac{(b+2 m) p+(a+2 m)}{p q-1} t} .
$$


where $C=C(n, m, t, s, a, b, p, q)$ is independent of $R>1$.

We now recall the following fundamental elliptic estimates. We shall apply these estimates frequently for the solutions of (1).

Lemma 2. (Sobolev inequalities on the sphere $S^{n-1}$ ) Let $n \geq 2$, integer $s \geq 1$ and $1<t<\tau \leq \infty$. For $z \in W^{s, t}\left(S^{n-1}\right)$, we have

$$
\|z\|_{L^{\tau}\left(S^{n-1}\right)} \leq C\left\|D_{\theta}^{s} z\right\|_{L^{t}\left(S^{n-1}\right)}+C\|z\|_{L^{1}\left(S^{n-1}\right)},
$$

where

$$
\begin{cases}\frac{1}{\tau}=\frac{1}{t}-\frac{s}{n-1}, & \text { if } s t+1<n \\ \tau=\infty, & \text { if } s t+1>n\end{cases}
$$

and $C=C(s, t, n, q)>0$ does not depend on $R>1$.

Lemma 3. (Elliptic $L^{\tau}$-estimate on $B_{R}$ ). Let $1<\tau<\infty$ and $m \geq 1$. For $z \in W^{2 m, \tau}\left(B_{2 R}\right)$, we have

$$
\int_{B_{R} \backslash B_{R / 2}}\left|D_{x}^{2 m} z\right|^{\tau} \leq C \int_{B_{2 R} \backslash B_{R / 4}}\left|\Delta_{x}^{m} z\right|^{\tau}+C R^{-2 m \tau} \int_{B_{2 R} \backslash B_{R / 4}}|z|^{\tau},
$$

where $C=C(n, m, \tau)>0$ does not depend on $R>1$.

LeMmA 4. (An interpolation inequality on $B_{R}$ ). Let $1 \leq \tau<\infty, m \geq 1$ and $1 \leq i \leq 2 m-1$. For sufficiently regular $z$, we have

$$
\int_{B_{R} \backslash B_{R / 2}}\left|D_{x}^{i} z\right|^{\tau} \leq C R^{(2 m-i) \tau} \int_{B_{2 R} \backslash B_{R / 4}}\left|\Delta_{x}^{m} z\right|^{\tau}+C R^{-i \tau} \int_{B_{2 R} \backslash B_{R / 4}}|z|^{\tau},
$$

where $C=C(n, m, \tau, i)>0$ does not depend on $R>1$.

By applying Lemma 1, Corollary 1, Lemma 3 and Lemma 4, we obtain the following estimates on the derivatives of $u$ and $v$.

Lemma 5. Let $m \geq 1$ and suppose that either $0 \leq i \leq 2 m-1$ and $\epsilon \geq 0$ or $i=2 m$ and $\epsilon>0$. Then, for a bounded nonnegative solution $(u, v)$ of (1) we have

$$
\begin{aligned}
& \int_{B_{2 R} \backslash B_{R}}\left|D_{x}^{i} u\right|^{1+\epsilon} \leq C R^{n-i-\frac{(b+2 m) p+(a+2 m)}{p q-1}+\epsilon(2 m-i+a)}, \\
& \int_{B_{2 R} \backslash B_{R}}\left|D_{x}^{i} v\right|^{1+\epsilon} \leq C R^{n-i-\frac{(a+2 m) q+(b+2 m)}{p q-1}+\epsilon(2 m-i+b)},
\end{aligned}
$$

where the constant $C=C(a, b, n, m, p, q, i, \epsilon)>0$ does not depend on $R>1$.

Proof. For the case $\epsilon \geq 0$ and $0 \leq i \leq 2 m-1$ we apply Lemma 1, Lemma 4 and Corollary 1 and for the case $\epsilon>0$ and $i=2 m$ we apply Lemma 1, Lemma 3 and 
Corollary 1 to get the following

$$
\begin{aligned}
& \int_{B_{2 R} \backslash B_{R}}\left|D_{x}^{i} u\right|^{1+\epsilon} \\
\leq & C R^{(2 m-i)(1+\epsilon)} \int_{B_{4 R} \backslash B_{R / 2}}\left|\Delta^{m} u\right|^{1+\epsilon}+C R^{-i(1+\epsilon)} \int_{B_{4 R} \backslash B_{R / 2}} u^{1+\epsilon} \\
\leq & C R^{(2 m-i)(1+\epsilon)+a \epsilon} \int_{B_{4 R} \backslash B_{R / 2}}|x|^{a} v^{p(1+\epsilon)}+C R^{-i(1+\epsilon)} \int_{B_{4 R} \backslash B_{R / 2}} u^{1+\epsilon} \\
\leq & C R^{(2 m-i)(1+\epsilon)+a \epsilon} \int_{B_{4 R} \backslash B_{R / 2}}|x|^{a} v^{p}+C R^{-i(1+\epsilon)} \int_{B_{4 R} \backslash B_{R / 2}} u \\
\leq & C R^{(2 m-i)(1+\epsilon)+a \epsilon} R^{n-2 m-\frac{(b+2 m) p+(a+2 m)}{p q-1}}+C R^{-i(1+\epsilon)} R^{n-\frac{(a+2 m) q+(b+2 m)}{p q-1}} \\
\leq & C R^{n-i-\frac{(b+2 m) p+(a+2 m)}{p q-1}+\epsilon(2 m+a-i)} .
\end{aligned}
$$

Note that we have used the boundedness assumption on $u$ and $v$ in the above when $\epsilon>0$. The proof of the other integral estimate on the gradients of $v$ is quite similar.

To prove our main results we apply the following Pohozaev identity.

Lemma 6. (Pohozaev identity). Suppose $\lambda, \gamma \in \mathbb{R}$ satisfy $\lambda+\gamma=n-2 m$. If $(u, v)$ is a nonnegative solution of (1), then it necessarily satisfy

1. For $m=2 k+1$ where $k \geq 0$,

$$
\begin{aligned}
& \left(\frac{n+a}{p+1}-\lambda\right) \int_{B_{R}}|x|^{a} v^{p+1}+\left(\frac{n+b}{q+1}-\gamma\right) \int_{B_{R}}|x|^{b} u^{q+1} \\
& =\frac{1}{p+1} \int_{\partial B_{R}}|x|^{a} v^{p+1} x \cdot \nu+\frac{1}{q+1} \int_{\partial B_{R}}|x|^{b} u^{q+1} x \cdot \nu-\int_{\partial B_{R}} \nabla \Delta^{k} u \cdot \nabla \Delta^{k} v x \cdot \nu \\
& +(\lambda+m-1) \int_{\partial B_{R}} \Delta^{k} v \partial_{\nu} \Delta^{k} u+(\gamma+m-1) \int_{\partial B_{R}} \Delta^{k} u \partial_{\nu} \Delta^{k} v \\
& +\int_{\partial B_{R}} \nabla \Delta^{k} u \cdot \nu x \cdot \nabla \Delta^{k} v+\int_{\partial B_{R}} \nabla \Delta^{k} v \cdot \nu x \cdot \nabla \Delta^{k} u \\
& +\lambda I(u, v)+\gamma I(v, u)+J(u, v)+J(v, u) .
\end{aligned}
$$

2. For $m=2 k$ where $k \geq 1$,

$$
\begin{aligned}
& \left(\frac{n+a}{p+1}-\lambda\right) \int_{B_{R}}|x|^{a} v^{p+1}+\left(\frac{n+b}{q+1}-\gamma\right) \int_{B_{R}}|x|^{b} u^{q+1} \\
& =\frac{1}{p+1} \int_{\partial B_{R}}|x|^{a} v^{p+1} x \cdot \nu+\frac{1}{q+1} \int_{\partial B_{R}}|x|^{b} u^{q+1} x \cdot \nu-\int_{\partial B_{R}} \Delta^{k} u \Delta^{k} v x \cdot \nu \\
& -\lambda I(u, v)-\gamma I(v, u)-J(u, v)-J(v, u),
\end{aligned}
$$

where

$$
\begin{aligned}
I(u, v) & :=\sum_{i=0}^{k-1} \int_{\partial B_{R}}\left(\Delta^{i} v \partial_{\nu} \Delta^{m-i-1} u-\Delta^{m-i-1} u \partial_{\nu} \Delta^{i} v\right) \\
J(u, v) & :=\sum_{i=0}^{k-1} \int_{\partial B_{R}}\left(\Delta^{i}(x \cdot \nabla v) \partial_{\nu} \Delta^{m-i-1} u-\Delta^{m-i-1} u \partial_{\nu} \Delta^{i}(x \cdot \nabla v)\right) .
\end{aligned}
$$


Proof. The proof is quite standard. We mention few technical facts that facilitates the computations. Suppose $z, w$ are smooth functions then for any $i \in \mathbf{N}$

$$
\begin{aligned}
\Delta^{i}(x \cdot \nabla z) & =2 i \Delta^{i} z+x \cdot \nabla \Delta^{i} z \\
\nabla z \cdot \nabla(x \cdot \nabla w)+\nabla w \cdot \nabla(x \cdot \nabla z) & =2 \nabla z \cdot \nabla w+x \cdot(\nabla z \cdot \nabla w) .
\end{aligned}
$$

Also for any $\lambda, \gamma \in \mathbb{R}$ the following equalities hold

$$
\begin{aligned}
(\lambda+\gamma) \int_{B_{R}} \nabla \Delta^{k} u \cdot \nabla \Delta^{k} v= & \lambda \int_{B_{R}}|x|^{a} v^{p+1}+\gamma \int_{B_{R}}|x|^{b} u^{q+1}+\lambda I(u, v)+\gamma I(v, u) \text { and } \\
(n-2 m) \int_{B_{R}} \nabla \Delta^{k} u \cdot \nabla \Delta^{k} v= & \int_{B_{R}}\left(\Delta^{m} u x \cdot \nabla v+\Delta^{m} v x \cdot \nabla u\right) \\
& +\int_{\partial B_{R}} \nabla \Delta^{k} u \cdot \nabla \Delta^{k} v x \cdot \nu-J(u, v)-J(v, u)
\end{aligned}
$$

when $m=2 k+1$ and for the case $m=2 k$ we have similar equations as

$$
\begin{aligned}
(\lambda+\gamma) \int_{B_{R}} \Delta^{k} u \Delta^{k} v= & \lambda \int_{B_{R}}|x|^{a} v^{p+1}+\gamma \int_{B_{R}}|x|^{b} u^{q+1}-\lambda I(u, v)-\gamma I(v, u) \text { and } \\
(n-2 m) \int_{B_{R}} \Delta^{k} u \Delta^{k} v= & -\int_{B_{R}}\left(\Delta^{m} u x \cdot \nabla v-\Delta^{m} v x \cdot \nabla u\right) \\
& +\int_{\partial B_{R}} \Delta^{k} u \Delta^{k} v x \cdot \nu+J(u, v)+J(v, u) .
\end{aligned}
$$

Finally for either $m=2 k$ or $m=2 k+1$ we have

$$
\begin{aligned}
& \int_{B_{R}}|x|^{a} v^{p} x \cdot \nabla v+\int_{B_{R}}|x|^{b} u^{q} x \cdot \nabla u \\
= & -\frac{n+a}{p+1} \int_{B_{R}}|x|^{a} v^{p+1}-\frac{n+b}{q+1} \int_{B_{R}}|x|^{b} u^{q+1} \\
& +\frac{1}{p+1} \int_{\partial B_{R}}|x|^{a} v^{p+1} x \cdot \nu+\frac{1}{q+1} \int_{\partial B_{R}}|x|^{b} u^{q+1} x \cdot \nu .
\end{aligned}
$$

We are now in the position to prove Theorem 1. The main technique here is to apply the Pohozaev identity that Lemma 6 and then taking the advantage of the elliptic regularity theory and in particular the lemmata mentioned before to get certain decay estimates on each boundary term appeared in the Pohozaev identity.

Proof of Theorem 1. Since $(p, q)$ satisfy (2), then we can choose $\lambda$ and $\gamma$ such that $\frac{n+a}{p+1}>\lambda$ and $\frac{n+b}{q+1}>\gamma$. Now, for all $R>1$ define the following positive function of $R$ that is in fact the left hand side of the Pohozaev identity

$$
L(R):=\left(\frac{n+a}{p+1}-\lambda\right) \int_{B_{R}}|x|^{a} v^{p+1}+\left(\frac{n+b}{q+1}-\gamma\right) \int_{B_{R}}|x|^{b} u^{q+1} .
$$

From Lemma 6 and for either $m=2 k$ or $m=2 k+1$ we have the following upper bound on $L$

$$
L(R) \leq C \sum_{i=1}^{3} U_{i}(R)
$$


where $C=C(n, m, p, q)$ is independent of $R$ and

$$
\begin{aligned}
U_{1}(R) & :=R^{n+a} \int_{S^{n-1}} v^{p+1}(R, \theta)+R^{n+b} \int_{S^{n-1}} u^{q+1}(R, \theta) \\
U_{2}(R) & :=R^{n-1} \sum_{j=0}^{m-1} \int_{S^{n-1}}\left(\left|D_{x}^{j} v(R, \theta)\right|\left|D_{x}^{2 m-j-1} u(R, \theta)\right|+\left|D_{x}^{j} u(R, \theta)\right|\left|D_{x}^{2 m-j-1} v(R, \theta)\right|\right) \\
U_{3}(R) & :=R^{n} \sum_{j=0}^{m-1} \int_{S^{n-1}}\left(\left|D_{x}^{j+1} v(R, \theta)\right|\left|D_{x}^{2 m-j-1} u(R, \theta)\right|+\left|D_{x}^{j+1} u(R, \theta)\right|\left|D_{x}^{2 m-j-1} v(R, \theta)\right|\right) .
\end{aligned}
$$

To get this upper bound we have used the following facts.

$$
\begin{aligned}
I(u, v) & \leq k \sum_{i=0}^{k-1} \int_{\partial B_{R}}\left(\left|D_{x}^{2 i} v\right|\left|D_{x}^{2 m-2 i-1} u\right|+\left|D_{x}^{2 m-2 i-2} u\right|\left|D_{x}^{2 i+1} v\right|\right) \\
& \leq k \sum_{j=0}^{2 k-1} \int_{\partial B_{R}}\left|D_{x}^{j} v \| D_{x}^{2 m-j-1} u\right| .
\end{aligned}
$$

Note that for any $i \in \mathbb{N}$ we have $\Delta^{i}(x \cdot \nabla v)=2 i \Delta^{i} v+x \cdot \nabla \Delta^{i} v$ and also $\nu \cdot \nabla\left(x \cdot \nabla \Delta^{i} v\right)=$ $\nu \cdot \nabla \Delta^{i} v+\sum_{s, t}^{n} x_{s} \nu_{t} \partial_{x_{s} x_{t}}\left(\Delta^{i} v\right)$. Using this we get

$$
\begin{aligned}
J(u, v) & \leq C I(u, v)+k \sum_{i=0}^{k-1} \int_{\partial B_{R}}\left(x \cdot \nabla \Delta^{i} v \partial_{\nu} \Delta^{m-i-1} u-\Delta^{m-i-1} u \partial_{\nu}\left(x \cdot \nabla \Delta^{i} v\right)\right) \\
& \leq C I(u, v)+k R \sum_{i=0}^{k-1} \int_{\partial B_{R}}\left(\left|D_{x}^{2 i+1} v\right|\left|D_{x}^{2 m-2 i-1} u\right|+\left|D_{x}^{2 m-2 i-2} u\right|\left|D_{x}^{2 i+2} v\right|\right) \\
& \leq C I(u, v)+k R \sum_{j=0}^{2 k-1} \int_{\partial B_{R}}\left|D_{x}^{j+1} v\right|\left|D_{x}^{2 m-j-1} u\right| .
\end{aligned}
$$

In what follows we find upper bounds on each $U_{i}$ when $1 \leq i \leq 3$. Let's first fix $\epsilon>0$ small enough now and then we pick the appropriate value later. Also, for the sake of simplicity of notations, throughout the proof, we use the notation $\|w\|_{t}$ to show the $L^{t}\left(S^{n-1}\right)$ estimates of $w(R, \theta)$ on the sphere that is $\|w\|_{L^{t}\left(S^{n-1}\right)}$ or $\left(\int_{S^{n-1}} w^{t}(R, \theta)\right)^{1 / t}$. Here are the upper bounds.

Upper bounds for $U_{1}$. Note that from Lemma 2 we have the Sobolev embedding $W^{2 m, 1+\epsilon}\left(S^{n-1}\right) \hookrightarrow L^{\infty}\left(S^{n-1}\right)$ in dimension $n=2 m+1$. Therefore,

$$
\begin{aligned}
\left(\int_{S^{n-1}} v^{p+1}(R, \theta)\right)^{\frac{1}{p+1}} & =\|v\|_{p+1} \leq\|v\|_{\infty} \leq C\left\|D_{\theta}^{2 m} v\right\|_{1+\epsilon}+C\|v\|_{1} \\
& \leq C R^{2 m}\left\|D_{x}^{2 m} v\right\|_{1+\epsilon}+C\|v\|_{1} .
\end{aligned}
$$

So, applying the same argument for $u$ we get

$$
\begin{aligned}
U_{1}(R) \leq & C R^{n+a}\left(R^{2 m}\left\|D_{x}^{2 m} v\right\|_{1+\epsilon}+\|v\|_{1}\right)^{p+1} \\
& +C R^{n+b}\left(R^{2 m}\left\|D_{x}^{2 m} u\right\|_{1+\epsilon}+\|u\|_{1}\right)^{q+1} .
\end{aligned}
$$

Upper bounds for $U_{2}$. For any $j=0, \cdots, m-1$ we have $1 \leq j+1 \leq m$ and also $1 \leq 2 m-1 \leq 2 m-j-1 \leq m$. So from Hölder's inequality we get

$$
\int_{S^{n-1}}\left|D_{x}^{j} v(R, \theta)\right|\left|D_{x}^{2 m-j-1} u(R, \theta)\right| \leq\left\|D_{x}^{j} v\right\|_{\frac{2 m}{j+1}}\left\|D_{x}^{2 m-j-1} u\right\|_{\frac{2 m}{2 m-j-1}} .
$$


Note that from Lemma 2 we get the embeddings $W^{2 m-j-1,1+\epsilon}\left(S^{n-1}\right) \hookrightarrow L^{\frac{2 m}{j+1}}\left(S^{n-1}\right)$ and $W^{j+1,1+\epsilon}\left(S^{n-1}\right) \hookrightarrow L^{\frac{2 m}{2 m-j-1}}\left(S^{n-1}\right)$ in dimension $n=2 m+1$. So,

$$
\left\|D_{x}^{j} v\right\|_{L \frac{2 m}{j+1}} \leq C\left\|D_{\theta}^{2 m-j-1} D_{x}^{j} v\right\|_{1+\epsilon}+C\left\|D_{x}^{j} v\right\|_{1} \leq C R^{2 m-j-1}\left\|D_{x}^{2 m-1} v\right\|_{1+\epsilon}+C\left\|D_{x}^{j} v\right\|_{1}
$$

and

$$
\begin{aligned}
\left\|D_{x}^{2 m-j-1} u\right\|_{L^{\frac{2 m}{2 m-j-1}}} & \leq C\left\|D_{\theta}^{j+1} D_{x}^{2 m-j-1} u\right\|_{1+\epsilon}+C\left\|D_{x}^{2 m-j-1} u\right\|_{1} \\
& \leq C R^{j+1}\left\|D_{x}^{2 m} u\right\|_{1+\epsilon}+C\left\|D_{x}^{2 m-j-1} u\right\|_{1}
\end{aligned}
$$

Therefore

$$
\begin{aligned}
U_{2}(R) \leq & C R^{n-1} \sum_{j=0}^{m-1}\left(R^{j+1}\left\|D_{x}^{2 m} u\right\|_{1+\epsilon}+\left\|D_{x}^{2 m-j-1} u\right\|_{1}\right) \\
& \times\left(R^{2 m-j-1}\left\|D_{x}^{2 m-1} v\right\|_{1+\epsilon}+\left\|D_{x}^{j} v\right\|_{1}\right) \\
& +C R^{n-1} \sum_{j=0}^{m-1}\left(R^{j+1}\left\|D_{x}^{2 m} v\right\|_{1+\epsilon}+\left\|D_{x}^{2 m-j-1} v\right\|_{1}\right) \\
& \times\left(R^{2 m-j-1}\left\|D_{x}^{2 m-1} u\right\|_{1+\epsilon}+\left\|D_{x}^{j} u\right\|_{1}\right) .
\end{aligned}
$$

Upper bounds for $U_{3}$. Similar arguments and embedding as for $U_{2}$ can be used for this term as well. Hölder's inequality yields

$$
\int_{S^{n-1}}\left|D_{x}^{j+1} v(R, \theta)\left\|D_{x}^{2 m-j-1} u(R, \theta) \mid \leq\right\| D_{x}^{j+1} v\left\|_{L^{\frac{2 m}{j+1}}}\right\| D_{x}^{2 m-j-1} u \|_{L^{\frac{2 m}{2 m-j-1}}} .\right.
$$

Again from the embeddings $W^{2 m-j-1,1+\epsilon}\left(S^{n-1}\right) \quad \hookrightarrow \quad L^{\frac{2 m}{j+1}}\left(S^{n-1}\right) \quad$ and $W^{j+1,1+\epsilon}\left(S^{n-1}\right) \hookrightarrow L^{\frac{2 m}{2 m-j-1}}\left(S^{n-1}\right)$ we get

$$
\begin{aligned}
\left\|D_{x}^{j+1} v\right\|_{L^{\frac{2 m}{j+1}}} & \leq C\left\|D_{\theta}^{2 m-j-1} D_{x}^{j+1} v\right\|_{1+\epsilon}+C\left\|D_{x}^{j+1} v\right\|_{1} \\
& \leq C R^{2 m-j-1}\left\|D_{x}^{2 m} v\right\|_{1+\epsilon}+C\left\|D_{x}^{j+1} v\right\|_{1}
\end{aligned}
$$

and

$$
\begin{aligned}
\left\|D_{x}^{2 m-j-1} u\right\|_{L^{\frac{2 m}{2 m-j-1}}} & \leq C\left\|D_{\theta}^{j+1} D_{x}^{2 m-j-1} u\right\|_{1+\epsilon}+C\left\|D_{x}^{2 m-j-1} u\right\|_{1} \\
& \leq C R^{j+1}\left\|D_{x}^{2 m} u\right\|_{1+\epsilon}+C\left\|D_{x}^{2 m-j-1} u\right\|_{1} .
\end{aligned}
$$

Therefore

$$
\begin{aligned}
U_{3}(R) \leq & C R^{n} \sum_{j=0}^{m-1}\left(R^{j+1}\left\|D_{x}^{2 m} u\right\|_{1+\epsilon}+\left\|D_{x}^{2 m-j-1} u\right\|_{1}\right) \\
& \times\left(R^{2 m-j-1}\left\|D_{x}^{2 m} v\right\|_{1+\epsilon}+\left\|D_{x}^{j+1} v\right\|_{1}\right) \\
& +C R^{n} \sum_{j=0}^{m-1}\left(R^{j+1}\left\|D_{x}^{2 m} v\right\|_{1+\epsilon}+\left\|D_{x}^{2 m-j-1} v\right\|_{1}\right) \\
& \times\left(R^{2 m-j-1}\left\|D_{x}^{2 m} u\right\|_{1+\epsilon}+\left\|D_{x}^{j+1} u\right\|_{1}\right) .
\end{aligned}
$$

Now we are ready to show that the upper bounds on each $U_{i}(R)$ converges to zero for an appropriate sequence of $R_{l}$ when $R_{l}$ converges to infinity. To construct 
such a sequence, for any $j=0, \cdots, 2 m-1$ and $i=2 m-1,2 m$ define the following sets where $M$ is a large constant that will be determined later.

$$
\begin{aligned}
\Gamma_{1}^{(j)}(R) & :=\left\{r \in(R / 2, R) ;\left\|D_{x}^{j} v\right\|_{1}>M R^{\left.-j-\frac{(a+2 m) q+(b+2 m)}{p q-1}\right\},}\right. \\
\Gamma_{2}^{(j)}(R) & :=\left\{r \in(R / 2, R) ;\left\|D_{x}^{j} u\right\|_{1}>M R^{\left.-j-\frac{(b+2 m) p+(a+2 m)}{p q-1}\right\},},\right. \\
\Gamma_{3}^{(i)}(R, \epsilon) & :=\left\{r \in(R / 2, R) ;\left\|D_{x}^{i} v\right\|_{1+\epsilon}^{1+\epsilon}>M R^{-i-\frac{(a+2 m) q+(b+2 m)}{p q-1}+\epsilon(2 m+b-i)}\right\}, \\
\Gamma_{4}^{(i)}(R, \epsilon) & :=\left\{r \in(R / 2, R) ;\left\|D_{x}^{i} u\right\|_{1+\epsilon}^{1+\epsilon}>M R^{-i-\frac{(b+2 m) p+(a+2 m)}{p q-1}+\epsilon(2 m+a-i)}\right\} .
\end{aligned}
$$

Note that from Lemma 5 for either $\epsilon \geq 0$ and $0 \leq t \leq 2 m-1$ or $\epsilon>0$ and $t=2 m$ we have

$$
\begin{aligned}
C & \geq R^{-n+t+\frac{(b+2 m) p+(a+2 m)}{p q-1}-\epsilon(2 m-t+a)} \int_{B_{R} \backslash B_{R / 2}}\left|D_{x}^{t} u\right|^{1+\epsilon} \\
& =R^{-n+t+\frac{(b+2 m) p+(a+2 m)}{p q-1}-\epsilon(2 m-t+a)} \int_{R / 2}^{R}\left\|D_{x}^{t} u\right\|_{1+\epsilon}^{1+\epsilon} r^{n-1} d r .
\end{aligned}
$$

So, for $i=2 m-1,2 m$ we have

$$
\begin{aligned}
C & \geq R^{-n+i+\frac{(b+2 m) p+(a+2 m)}{p q-1}-\epsilon(2 m-i+a)} \int_{\Gamma_{4}^{(i)}(R, \epsilon)}\left\|D_{x}^{i} u\right\|_{1+\epsilon}^{1+\epsilon} r^{n-1} d r \\
& \geq M R^{-n+i+\frac{(b+2 m) p+(a+2 m)}{p q-1}-\epsilon(2 m-i+a)}\left|\Gamma_{4}^{(i)}(R, \epsilon)\right| R^{n-1} R^{-i-\frac{(b+2 m) p+(a+2 m)}{p q-1}+\epsilon(2 m+a-i)} \\
& =M\left|\Gamma_{4}^{(i)}(R, \epsilon)\right| R^{-1}
\end{aligned}
$$

that is $\left|\Gamma_{4}^{(i)}(R, \epsilon)\right| \leq \frac{C R}{M}$. Similarly one can apply the same argument to show that $\left|\Gamma_{3}^{(i)}(R, \epsilon)\right| \leq \frac{C R}{M},\left|\Gamma_{1}^{(j)}(R)\right| \leq \frac{C R}{M}$ and $\left|\Gamma_{2}^{(j)}(R)\right| \leq \frac{C R}{M}$. Therefore, we can choose $M$ large enough to make sure that

$$
\sum_{\tilde{i}=3}^{4} \sum_{i=2 m-1}^{2 m}\left|\Gamma_{\tilde{i}}^{(i)}(R, \epsilon)\right|+\sum_{\tilde{j}=1}^{2} \sum_{j=0}^{2 m-1}\left|\Gamma_{\tilde{j}}^{(j)}(R)\right| \leq \frac{(4 m+4) C R}{M} \leq \frac{R}{3} .
$$

Hence, for each $R \geq 1$, we can find

$$
R_{l} \in(R / 2, R) \backslash\left\{\cup_{i=2 m-1}^{2 m} \cup_{\tilde{i}=3}^{4} \Gamma_{\tilde{i}}^{(i)}(R, \epsilon), \cup_{j=0}^{2 m-1} \cup_{\tilde{j}=1}^{2} \Gamma_{\tilde{j}}^{(j)}(R)\right\} \neq \phi .
$$

Now we use the sequence $R_{l}$ to get a decay estimate on each $U_{i}(R)$ where $1 \leq i \leq 3$.

Decay estimate on $U_{1}$. From (12) we get

$$
\begin{aligned}
U_{1}\left(R_{l}\right) \leq & C R_{l}^{n+a}\left(R_{l}^{2 m} R_{l}^{\left(-2 m-\frac{(a+2 m) q+(b+2 m)}{p q-1}+\epsilon b\right) \frac{1}{1+\epsilon}}+R_{l}^{-\frac{(a+2 m) q+(b+2 m)}{p q-1}}\right)^{p+1} \\
& +C R_{l}^{n+b}\left(R_{l}^{2 m} R_{l}^{\left(-2 m-\frac{(b+2 m) p+(a+2 m)}{p q-1}+\epsilon a\right) \frac{1}{1+\epsilon}}+R_{l}^{-\frac{(b+2 m) p+(a+2 m)}{p q-1}}\right)^{q+1} \\
\leq & C\left(R_{l}^{-f_{1}(\epsilon)}+R_{l}^{-\tilde{f}_{1}(\epsilon)}\right),
\end{aligned}
$$

where

$$
\begin{aligned}
& f_{1}(\epsilon)=(p+1)\left[\left(2 m+\frac{(a+2 m) q+(b+2 m)}{p q-1}-b \epsilon\right) \frac{1}{1+\epsilon}-2 m-\frac{n+a}{p+1}\right], \\
& \tilde{f}_{1}(\epsilon)=(q+1)\left[\left(2 m+\frac{(b+2 m) p+(a+2 m)}{p q-1}-a \epsilon\right) \frac{1}{1+\epsilon}-2 m-\frac{n+b}{q+1}\right] .
\end{aligned}
$$


Decay estimate on $U_{2}$. From (13) we get

$$
\begin{aligned}
& U_{2}\left(R_{l}\right) \leq C R_{l}^{n-1} \sum_{j=0}^{m-1}\left(R_{l}^{j+1} R_{l}^{\left(-2 m-\frac{(b+2 m) p+(a+2 m)}{p q-1}+\epsilon a\right) \frac{1}{1+\epsilon}}+R_{l}^{-2 m+j+1-\frac{(b+2 m) p+(a+2 m)}{p q-1}}\right) \\
& \left(R_{l}^{2 m-j-1} R_{l}^{\left(-2 m+1-\frac{(a+2 m) q+(b+2 m)}{p q-1}+\epsilon(b+1)\right) \frac{1}{1+\epsilon}}+R_{l}^{-j-\frac{(a+2 m) q+(b+2 m)}{p q-1}}\right) \\
& +C R_{l}^{n-1} \sum_{j=0}^{m-1}\left(R_{l}^{j+1} R_{l}^{\left(-2 m-\frac{(a+2 m) q+(b+2 m)}{p q-1}+\epsilon b\right) \frac{1}{1+\epsilon}}+R_{l}^{-2 m+j+1-\frac{(a+2 m) q+(b+2 m)}{p q-1}}\right) \\
& \left(R_{l}^{2 m-j-1} R_{l}^{\left(-2 m+1-\frac{(b+2 m) p+(a+2 m)}{p q-1}+\epsilon(a+1)\right) \frac{1}{1+\epsilon}}+R_{l}^{-j-\frac{(b+2 m) p+(a+2 m)}{p q-1}}\right) \\
& =2 m C R_{l}^{n+2 m}\left(R_{l}^{\left(-2 m-\frac{(b+2 m) p+(a+2 m)}{p q-1}+\epsilon a\right) \frac{1}{1+\epsilon}}+R_{l}^{-2 m-\frac{(b+2 m) p+(a+2 m)}{p q-1}}\right) \\
& \left(R_{l}^{\left(-2 m-\frac{(a+2 m) q+(b+2 m)}{p q-1}+\epsilon b\right) \frac{1}{1+\epsilon}}+R_{l}^{-2 m-\frac{(a+2 m) q+(b+2 m)}{p q-1}}\right) \\
& \leq C R_{l}^{-f_{2}(\epsilon)}
\end{aligned}
$$

where $f_{2}(\epsilon)$ is defined as

$$
f_{2}(\epsilon):=-n+2 m\left(\frac{1-\epsilon}{1+\epsilon}\right)+\frac{(b+2 m)(p+1)+(a+2 m)(q+1)}{(p q-1)(1+\epsilon)}-\frac{(a+b) \epsilon}{1+\epsilon}
$$

Similarly, from (14) one can show that

$$
U_{3}\left(R_{l}\right) \leq C R_{l}^{-f_{2}(\epsilon)} .
$$

From (28) and the upper bounds on each $U_{i}$ we have

$$
L\left(R_{l}\right) \leq C \sum_{i=1}^{3} U_{i}\left(R_{l}\right) \leq C\left(R_{l}^{-f_{2}(\epsilon)}+R_{l}^{-f_{1}(\epsilon)}+R_{l}^{-\tilde{f}_{1}(\epsilon)}\right) .
$$

For each $\epsilon \geq 0$ define $f(\epsilon):=\min \left\{f_{1}(\epsilon), \tilde{f}_{1}(\epsilon), f_{2}(\epsilon)\right\}$. So, $L\left(R_{l}\right) \leq C R_{l}^{-f(\epsilon)}$. Now to finish the proof we show that for $\epsilon>0$ small enough $f(\epsilon)>0$. Note that by a straightforward calculation, one can see that $\frac{n+a}{p+1}+\frac{n+b}{q+1}>n-2 m$ is equivalent to each one of the following inequalities.

$$
\begin{aligned}
& f_{2}(0)=-n+2 m+\frac{(b+2 m)(p+1)+(a+2 m)(q+1)}{p q-1}>0 \\
& f_{1}(0)=(p+1)\left(\frac{(a+2 m) q+(b+2 m)}{p q-1}-\frac{n+a}{p+1}\right)>0 \\
& \tilde{f}_{1}(0)=(q+1)\left(\frac{(b+2 m) p+(a+2 m)}{p q-1}-\frac{n+b}{q+1}\right)>0 .
\end{aligned}
$$

Therefore, we can choose $\epsilon>0$ small enough such that $f(\epsilon)>0$. We now conclude by sending $R \rightarrow \infty$ that $L\left(R_{l}\right)=0$ and then $u=v=0$.

3. Liouville theorems for radial solutions via ODE arguments. In this section we focus on the radial solutions of (1) and we prove Theorem 2. When we 
are dealing with radial solutions, the weight functions $|x|^{a}$ and $|x|^{b}$ would not change the level of difficulty of the problem much. In other words, the methods and ideas that are used for the case $a=b=0$, can be directly adjusted. Therefore, we omit some of the proofs. What we would like to emphasize in this section is how the radial assumption make it easier to get decay estimates on solutions of (1), see Lemma 8 and Corollary 2. Since we do not need to apply Sobolev embeddings and regularity theory, there will be no restriction on the dimension. The methods that we apply here are strongly motivated by the methods used in $[16,15]$.

Lemma 7. Suppose that $(u, v)$ is a positive solution of (1), then $(-\Delta)^{i} u>0$ and $(-\Delta)^{i} v>0$ where $i=1,2, \cdots, m$.

Proof. The proof directly follows the methods given in [29] for polyharmonic equations that is also used in [14] for polyharmonic systems. The idea is to define the average function on $\partial B_{R}$ as it is defined in [18].

Lemma 8. Let $n \geq 3$. Suppose that $(u, v)$ is a positive radial solution of (1). Then the following pointwise decay estimates hold for any $i=0, \cdots, m$ and $j=0, \cdots, m-1$ provided $r>0$

$$
\begin{aligned}
(-\Delta)^{i} u(r) & \leq C_{n, m, i} r^{-2 i-\frac{(b+2 m) p+(a+2 m)}{p q-1}} \\
(-\Delta)^{i} v(r) & \leq C_{n, m, i} r^{-2 i-\frac{(a+2 m) q+(b+2 m)}{p q-1}} \\
\left|\Delta^{j} u^{\prime}(r)\right| & \leq C_{n, m, j} r^{-2 j-1-\frac{(b+2 m) p+(a+2 m)}{p q-1}} \\
\left|\Delta^{j} v^{\prime}(r)\right| & \leq C_{n, m, j} r^{-2 j-1-\frac{(a+2 m) q+(b+2 m)}{p q-1}} .
\end{aligned}
$$

Proof. Define $u_{i}=(-\Delta)^{i} u$ where $i=0, \cdots, m-1$. From Lemma 7 we have $u_{i}>0$ and $-\Delta u_{i}>0$. Therefore, $u_{i}^{\prime}<0$. Note that from the definition of the sequences $\left(u_{i}\right)_{i}$ we have $u_{i+1}=-\Delta u_{i}$ when $i=0, \cdots, m-1$ and $r>0$

$$
\begin{aligned}
-u_{i}^{\prime}(r) r^{n-1} & =\frac{r^{n}}{n} u_{i+1}(r)-\frac{1}{n} \int_{0}^{r} u_{i+1}^{\prime}(s) s^{n} d s \\
& \geq \frac{r^{n}}{n} u_{i+1}(r)
\end{aligned}
$$

that is $r u_{i+1}(r) \leq-n u_{i}^{\prime}(r)$. On the other hand, since $u_{i}>0$ and $-\Delta u_{i}>0$ we have $r u_{i}^{\prime}+(n-2) u_{i} \geq 0$ that is $-r u_{i}^{\prime} \leq(n-2) u_{i}$. Therefore,

$$
u_{i+1}(r) \leq\left(\frac{n(n-2)}{r^{2}}\right)^{i+1} u \text { for all } i=0, \cdots, m-1 .
$$

In particular, $u_{m}(r) \leq\left(\frac{n(n-2)}{r^{2}}\right)^{m} u(r)$ and note that $u_{m}(r)=(-\Delta)^{m} u(r)=r^{a} v^{p}(r)$. Therefore,

$$
v^{p}(r) \leq(n(n-2))^{m} r^{-2 m-a} u(r)
$$

Similarly, for $v$ we get

$$
u^{q}(r) \leq(n(n-2))^{m} r^{-2 m-b} v(r) .
$$


From (23) and (22) we get

$$
\begin{aligned}
& u(r) \leq(n(n-2))^{m\left(\frac{p+1}{p q-1}\right)} r^{-\frac{(a+2 m)+(b+2 m) p}{p q-1}} \\
& v(r) \leq(n(n-2))^{m\left(\frac{q+1}{p q-1}\right)} r^{-\frac{(b+2 m)+(a+2 m) q}{p q-1}} .
\end{aligned}
$$

Then from (21) and (23) we have for all $i=0, \cdots, m$

$$
\begin{aligned}
& u_{i}=(-\Delta)^{i} u(r) \leq(n(n-2))^{i+m\left(\frac{p+1}{p q-1}\right)} r^{-2 i-\frac{(a+2 m)+(b+2 m) p}{p q-1}} \\
& v_{i}=(-\Delta)^{i} v(r) \leq(n(n-2))^{i+m\left(\frac{q+1}{p q-1}\right)} r^{-2 i-\frac{(b+2 m)+(a+2 m) q}{p q-1}} .
\end{aligned}
$$

To get the other bounds on the derivative of $u_{i}$ and $v_{i}$ one can use $0 \leq-r u_{i}^{\prime} \leq(n-2) u_{i}$ where $i=0, \cdots, m-1$.

Corollary 2. Let $n \geq 2 m+1$ for $m \geq 1$. Suppose that $(u, v)$ is a positive radial solution of (1) and (2) holds. Then for any $t, \tilde{t}=0, \cdots, m$ and $s, \tilde{s}=0, \cdots, m-1$ when $R \rightarrow \infty$

$$
\begin{aligned}
& R^{n}\left|\Delta^{t} u(R)\right|\left|\Delta^{\tilde{t}} v(R)\right| \rightarrow 0 \text { where } t+\tilde{t}=m \\
& R^{n-1}\left|\Delta^{t} u(R)\right|\left|\Delta^{s} v^{\prime}(R)\right| \rightarrow 0 \text { where } t+s=m-1 \\
& R^{n-1}\left|\Delta^{t} v(R)\right|\left|\Delta^{s} u^{\prime}(R)\right| \rightarrow 0 \text { where } t+s=m-1 \\
& R^{n}\left|\Delta^{s} u^{\prime}(R)\right|\left|\Delta^{\tilde{s}} v^{\prime}(R)\right| \rightarrow 0 \text { where } s+\tilde{s}=m-1 \text {. }
\end{aligned}
$$

Proof of Theorem 2. The idea is to apply the Pohozaev identity as in the proof of Theorem 1. Since $(p, q)$ satisfy (2), then we can choose $\lambda$ and $\gamma$ such that $\frac{n+a}{p+1}>\lambda$ and $\frac{n+b}{q+1}>\gamma$. Now, for all $R>1$ define the following positive function of $R$ that is the left hand side of the Pohozaev identity

$$
L(R):=\left(\frac{n+a}{p+1}-\lambda\right) \int_{B_{R}}|x|^{a} v^{p+1}+\left(\frac{n+b}{q+1}-\gamma\right) \int_{B_{R}}|x|^{b} u^{q+1} .
$$

From Lemma 6 and for either $m=2 k$ or $m=2 k+1$ it is straightforward to observe that the following upper bound on $L$ holds

$$
L(R) \leq C \sum_{i=1}^{5} U_{i}(R)
$$

where $C=C(m, n, a, b, p, q)$ is independent of $R$ and

$$
\begin{aligned}
& U_{1}(R):=R^{n+a} v^{p+1}(R)+R^{n+b} u^{q+1}(R) \\
& U_{2}(R):=R^{n-1} \sum_{j=0}^{k}\left(\left|\Delta^{j} v(R)\right|\left|\Delta^{m-j-1} u^{\prime}(R)\right|+\left|\Delta^{m-j-1} u(R)\right|\left|\Delta^{j} v^{\prime}(R)\right|\right) \\
& U_{3}(R):=R^{n-1} \sum_{j=0}^{k}\left(\left|\Delta^{j} u(R)\right|\left|\Delta^{m-j-1} v^{\prime}(R)\right|+\left|\Delta^{m-j-1} v(R)\right|\left|\Delta^{j} u^{\prime}(R)\right|\right) \\
& U_{4}(R):=R^{n} \sum_{j=0}^{k}\left|\Delta^{j} v^{\prime}(R)\right|\left|\Delta^{m-j-1} u^{\prime}(R)\right|+R^{n} \sum_{j=0}^{k-1}\left|\Delta^{m-j-1} u(R)\right|\left|\Delta^{j+1} v(R)\right| \\
& U_{5}(R):=R^{n} \sum_{j=0}^{k}\left|\Delta^{j} u^{\prime}(R)\left\|\Delta^{m-j-1} v^{\prime}(R)\left|+R^{n} \sum_{j=0}^{k-1}\right| \Delta^{m-j-1} v(R)\right\| \Delta^{j+1} u(R)\right| .
\end{aligned}
$$


Not that to get this upper bound we have used the following facts. Suppose $w, z$ are radial functions defined on a ball then $\partial_{\nu} w=\partial_{r} w, \nabla w \cdot \nabla z=w_{r} z_{r}$ and $x \cdot \nabla w=r w_{r}$. Therefore, for any $R>1$

$$
\begin{aligned}
\frac{1}{p+1} \int_{\partial B_{R}}|x|^{a} v^{p+1} x \cdot \nu & \leq C R^{n+a} v^{p+1}(R) \\
\frac{1}{q+1} \int_{\partial B_{R}}|x|^{b} u^{q+1} x \cdot \nu & \leq C R^{n+b} u^{q+1}(R) \\
\int_{\partial B_{R}} \nabla \Delta^{k} u \cdot \nabla \Delta^{k} v x \cdot \nu & \leq C R^{n}\left|\Delta^{k} v^{\prime}(R)\right|\left|\Delta^{k} u^{\prime}(R)\right| \\
\int_{\partial B_{R}} \Delta^{k} v \partial_{\nu} \Delta^{k} u & \leq C R^{n-1}\left|\Delta^{k} v(R)\right|\left|\Delta^{k} u^{\prime}(R)\right| \\
\int_{\partial B_{R}} \nabla \Delta^{k} u \cdot \nu x \cdot \nabla \Delta^{k} v & \leq C R^{n}\left|\Delta^{k} v^{\prime}(R)\right|\left|\Delta^{k} u^{\prime}(R)\right| \\
I(u, v) & \leq C R^{n-1} \sum_{j=0}^{k-1}\left(\left|\Delta^{j} v(R)\right|\left|\Delta^{m-j-1} u^{\prime}(R)\right|+\left|\Delta^{m-j-1} u(R)\right|\left|\Delta^{j} v^{\prime}(R)\right|\right) .
\end{aligned}
$$

To find an upper bound on $J(u, v)$ we apply the fact that $\Delta^{j}(x \cdot \nabla v)=2 j \Delta^{j} v+x \cdot \nabla \Delta^{j} v$ and then

$$
\begin{aligned}
J(u, v) \leq & C_{m} I(u, v)+\sum_{j=0}^{k-1} \int_{\partial B_{R}}\left(\left(x \cdot \nabla \Delta^{j} v\right) \partial_{\nu} \Delta^{m-j-1} u-\Delta^{m-j-1} u \partial_{\nu}\left(x \cdot \nabla \Delta^{j} v\right)\right) \\
\leq & C_{m} I(u, v)+R^{n} \sum_{j=0}^{k-1}\left|\Delta^{j} v^{\prime}(R)\left\|\Delta^{m-j-1} u^{\prime}(R)\left|+C R^{n} \sum_{j=0}^{k-1}\right| \Delta^{m-j-1} u(R)\right\| \Delta^{j+1} v(R)\right| \\
& +C R^{n-1} \sum_{j=0}^{k-1}\left|\Delta^{m-j-1} u(R) \| \Delta^{j} v^{\prime}(R)\right| .
\end{aligned}
$$

Note that in the above we have also used the fact that

$$
\begin{aligned}
\partial_{\nu}\left(x \cdot \nabla \Delta^{j} v\right) & =\left(R \Delta^{j} v^{\prime}(R)\right)^{\prime}=R \Delta^{j} v^{\prime \prime}(R)+\Delta^{j} v^{\prime}(R) \\
& =R \Delta^{j} v^{\prime \prime}(R)+(n-1) \Delta^{j} v^{\prime}(R)-(n-2) \Delta^{j} v^{\prime}(R) \\
& =R \Delta^{j+1} v(R)-(n-2) \Delta^{j} v^{\prime}(R) \\
& \leq C R\left|\Delta^{j+1} v(R)\right|+C\left|\Delta^{j} v^{\prime}(R)\right| .
\end{aligned}
$$

In what follows we apply Corollary 2 to show that $L(R) \rightarrow 0$ as $R \rightarrow \infty$. Note that $U_{1}(R) \leq C R^{n} v\left|\Delta^{m} u\right|+C R^{n} u\left|\Delta^{m} v\right|$. Then applying (24) when $t=m$ and $\tilde{t}=0$ and also when $t=0$ and $\tilde{t}=m$, we get $U_{1}(R) \rightarrow 0$ as $R \rightarrow \infty$. From the decay estimates (26) and (25) when $t$ and $s$ are set to be $0 \leq j \leq k$ and $0 \leq m-k-1 \leq m-j-1 \leq m-1$ we get $U_{2}(R)$ and $U_{3}(R) \rightarrow 0$ as $R \rightarrow \infty$. Similarly, from the decay estimates (24) and (27) we get $U_{4}(R)$ and $U_{5}(R) \rightarrow 0$ as $R \rightarrow \infty$. Therefore,

$$
L(R) \rightarrow 0 \text { as } R \rightarrow \infty
$$

On the other hand, multiplying both equations of (1) with $v$ and $u$ we have

$$
\begin{aligned}
\int_{B_{R}}|x|^{a} v^{p+1} & =\int_{B_{R}} v(-\Delta)^{m} u \\
\int_{B_{R}}|x|^{b} v^{q+1} & =\int_{B_{R}} u(-\Delta)^{m} v
\end{aligned}
$$


and

$$
\begin{aligned}
\int_{B_{R}} v \Delta^{m} u-\int_{B_{R}} u \Delta^{m} v & =\sum_{i=0}^{m-1} \int_{\partial B_{R}}\left(\Delta^{i} v \partial_{\nu} \Delta^{m-i-1} u-\Delta^{m-i-1} u \partial_{\nu} \Delta^{i} v\right) \\
& \leq C R^{n-1} \sum_{i=0}^{m-1}\left|\Delta^{i} v\right|\left|\Delta^{m-i-1} u^{\prime}(R)\right|+\left|\Delta^{m-i-1} u(R)\right|\left|\Delta^{i} v^{\prime}(R)\right| .
\end{aligned}
$$

Now applying Corollary 2 and in fact the decay estimates (26) when $t=i$ and $s=$ $m-i-1$ and also (25) when $t=m-i-1$ and $s=i$ we get $\int_{B_{R}} v \Delta^{m} u-\int_{B_{R}} u \Delta^{m} v \rightarrow 0$ as $R \rightarrow \infty$. Therefore,

$$
\int_{B_{R}}|x|^{a} v^{p+1}-\int_{B_{R}}|x|^{b} v^{q+1} \rightarrow 0 \text { as } R \rightarrow \infty .
$$

From this, (29) and the fact that $\lambda+\gamma=n-2 m$ we get the following as $R \rightarrow \infty$

$$
\begin{aligned}
& \left(\frac{n+a}{p+1}+\frac{n+b}{q+1}-(n-2 m)\right) \int_{B_{R}}|x|^{a} v^{p+1} \rightarrow 0 \\
& \left(\frac{n+b}{q+1}+\frac{n+a}{p+1}-(n-2 m)\right) \int_{B_{R}}|x|^{b} u^{q+1} \rightarrow 0 .
\end{aligned}
$$

From this and the fact that (2) holds we conclude that $u=v=0$.

\section{REFERENCES}

[1] J. Busca And R. Manásevich, A Liouville-type theorem for Lane-Emden systems, Indiana Univ. Math. J., 51 (2002), pp. 37-51.

[2] L. A. Caffarelli, B. Gidas, And J. Spruck, Asymptotic symmetry and local behavior of semilinear elliptic equations with critical Sobolev growth, Comm. Pure Appl. Math., 42:3 (1989), pp. 271-297.

[3] G. Caristi, L. D'Ambrosio, And E. Mitidieri, Representation formulae for solutions to some classes of higher order systems and related Liouville theorems, Milan Journal of Mathematics, 76 (2008), pp. 27-67.

[4] W. X. CHEN AND C. LI, Classification of solutions of some nonlinear elliptic equations, Duke Math. J., 63:3 (1991), pp. 615-622.

[5] C. Cowan, A Liouville theorem for a fourth order Hénon equation, preprint. http://arxiv.org/abs/1110.2246

[6] M. Fazly and N. Ghoussoub, On the Hénon-Lane-Emden conjecture, Disc. Cont. Dyn. Syst. A, 34:6 (2014), pp. 2513-2533.

[7] P. Felmer And D. G. DE Figueiredo, A Liouville-type theorem for elliptic systems, Ann. Sc. Norm. Super. Pisa XXI (1994), pp. 259-284.

[8] B. Gidas, W. M. Ni, AND L. Nirenberg, Symmetry of positive solutions of nonlinear elliptic equations in $\mathbb{R}^{N}$, Mathematical analysis and applications, Part A, pp. 369-402, Adv. in Math. Suppl. Stud., 7a, Academic Press, New York-London, 1981.

[9] B. Gidas AND J. SpRUCK, Global and local behavior of positive solutions of nonlinear elliptic equations, Commun. Pure Appl. Math., 34 (1981), pp. 525-598.

[10] B. Gidas and J. Spruck, A priori bounds for positive solutions of nonlinear elliptic equations, Comm. Partial Differential Equations, 6:8 (1981), pp. 883-901.

[11] M. HÉnon, Numerical experiments on the stability of spherical stellar systems, Astron. Astrophys, 24 (1973), pp. 229-238.

[12] T. LEI, Asymptotic properties of positive solutions of the Hardy-Sobolev type equations, Journal of Differential Equations, 254 (2013), pp. 1774-1799.

[13] C. S. LiN, A classification of solutions of a conformally invariant fourth order equation in $\mathbb{R}^{N}$, Comment. Math. Helv., 73 (1998), pp. 206-231.

[14] J. LiU, Y. Guo, AND Y. Zhang, Liouville-type theorems for polyharmonic systems in $\mathbf{R}^{n}$, J. Differential Equations, 225:2 (2006), pp. 685-709. 
[15] E. MitidiERI, Nonexistence of positive solutions of semilinear elliptic systems in $\mathbb{R}^{N}$, Differential Integral Equations, 9 (1996), pp. 465-479.

[16] E. Mitidieri, A Rellich type identity and applications, Comm. Partial Differential Equations, 18 (1993), no. 1-2, pp. 125-151.

[17] E. Mitidieri and S. I. Pokhozhaev, A priori estimates and the absence of solutions of nonlinear partial differential equations and inequalities, Tr. Mat. Inst. Steklova, 234 (2001), pp. 1-384.

[18] W. M. NI, A nonlinear Dirichlet problem on the unit ball and its applications, Indiana Univ. Math. J., 31 (1982), pp. 801-807.

[19] Q. H. Phan, Liouville-type theorems and bounds of solutions for Hardy-Hénon elliptic systems, Adv. Diff. Equ., 17 (2012), pp. 605-634.

[20] Q. H. Phan AND Ph. Souplet, Liouville-type theorems and bounds of solutions of Hardy-Hénon equations, J. Diff. Equ., 252 (2012), pp. 2544-2562.

[21] P. Poláčik, P. Quittner, And Ph. Souplet, Singularity and decay estimates in superlinear problems via Liouville-type theorems, Part I: Elliptic systems, Duke Math. J., 139 (2007), pp. 555-579.

[22] P. Quittner And Ph. Souplet, Superlinear Parabolic Problems. Blow-Up, Global Existence and Steady States, Birkhauser Verlag, Basel, 2007.

[23] J. Serrin AND H. Zou, Non-existence of positive solutions of Lane-Emden systems, Differential Integral Equations, 9 (1996), pp. 635-653.

[24] J. Serrin And H. Zou, Existence of positive solutions of the Lane-Emden system, Atti Semin. Mat. Fis. Univ.Modena, 46 (1998), pp. 369-380.

[25] D. Smets, J. Su, And M. Willem, Non radial ground states for the Hénon equation, Commun. Contemp. Math., 4:3 (2002), pp. 467-480.

[26] Рн. Souplet, The proof of the Lane-Emden conjecture in four space dimensions, Adv. Math., 221 (2009), pp. 1409-1427.

[27] M. A. S. Souto, A priori estimates and existence of positive solutions of non-linear cooperative elliptic systems, Differential Integral Equations, 8 (1995), pp. 1245-1258.

[28] X. YAn, A Liouville-type theorem for higher order elliptic systems, J. Math. Anal. Appl., 387:1 (2012), pp. 153-165.

[29] J. WEI AND X. XU, Classification of solutions of higher order conformally invariant equations, Math. Ann., 313:2 (1999), pp. 207-228. 
